\title{
Aspectos preventivos en migraña y trabajo. Encuesta europea
}

Preventive Aspects for Migraine and the Workplace: A European Survey

\author{
María Teófila Vicente Herrero ${ }^{1}$ \\ $M^{a}$ Victoria Ramírez Iñiguez de la Torre ${ }^{2}$ \\ Luis Reinoso Barbero ${ }^{3}$ \\ Elena Ruiz de la Torre ${ }^{4}$ \\ ${ }^{1}$ Grupo Correos, Valencia, España. \\ ${ }^{2}$ Grupo Correos, Albacete, España. \\ ${ }^{3}$ Banco Santander, Madrid, España. \\ ^European Migraine Headache Alliance, Bruselas, Bélgica.
}

Fechas · Dates

Recibido: 2020.04 .21

Aceptado: 2020.12 .18

Publicado: 2021.01.21
Correspondencia · Corresponding Author

María Teófila Vicente Herrero.

Especialista en Medicina del Trabajo, Grupo Correos, Valencia, España.

vicenteherreromt@gmail.com 


\title{
Resumen
}

Introducción: La promoción de la salud en el trabajo integra las iniciativas en salud y seguridad en ámbito ocupacional, con mejoras personales, incremento de productividad y menores riesgos y gastos sociales, especialmente en migraña, como enfermedad neurológica con prevalencia estimada en el $11 \%$ de la población. El objetivo de este trabajo fue conocer las condiciones preventivas de los trabajadores con migraña y las opciones de gestión preventiva en sus empresas.

Método: Estudio observacional transversal realizado en 3.342 pacientes de España, Italia, Francia, Portugal, Irlanda, Reino Unido, Alemania y otros países de la UE mediante encuesta anónima en la web de la European Migraine \& Headache Alliance (EMHA-web), entre septiembre de 2018 y enero de 2019.

Resultados: Estrés laboral (77.65\%) y uso de PVD (63.87\%) son los riesgos más referidos por los trabajadores con migraña. El $43.71 \%$ de trabajadores desconoce el tipo de Servicio de Prevención de su empresa, 49.06\% no dispone de servicio médico; el 67.67\% no ha tenido impedimentos de acceso laboral por migraña, ni despido o no renovación del contrato (88,29\%), pero el $42,14 \%$ tuvo algún conflicto por pérdida de productividad; el $26,54 \%$ desconoce el concepto de especial sensibilidad o no lo ha solicitado por migraña $(63,8 \%)$, ni ha demandado modificaciones laborales $(67,64 \%)$ o cambio de puesto de trabajo $(80,89 \%)$; un $55,42 \%$ no se ha sentido comprendido ni apoyado por su empresa en sus limitaciones por migraña, pero sí por los compañeros (63,07\%)

Conclusión: Se observa una deficiente información preventiva y escaso uso de las opciones de gestión adaptativa en las empresas para personas con migraña.

Palabras clave: migraña, prevención de riesgos laborales, salud laboral

\begin{abstract}
Introduction: Workplace health promotion integrates initiatives in health and safety in the occupational field, with personal improvements, increased productivity and lower risks and social cost, especially with respect to migraine headaches, a neurological disorder affecting approximately $11 \%$ of the population. The objective of this study was to know the preventive resources available to workers with migraine headaches and the preventive management options in their companies.
\end{abstract}

Method: Cross-sectional observational study of 3,342 patients from Spain, Italy, France, Portugal, Ireland, United Kingdom, Germany and other European Union countries, conducted through an anonymous survey on the web of the European Migraine \& Headache Alliance (EMHA-web), from September 2018 to January 2019.

Results: Occupational stress (77.65\%) and use of computer monitors (63.87\%) are the most common risks described by workers with migraine. About. $43.71 \%$ of workers are not familiar with the type of occupational health service present in their company, $49.06 \%$ do not have a medical service; $67.67 \%$ reported no work-related limitations due to migraine, neither dismissal nor non-renewal of their contract (88.29\%), but $42.14 \%$ had experienced some conflict due to decreased productivity; $26.54 \%$ were unaware of the concept of vulnerable workers or had not requested this status because of their migraine (63.8\%), nor had 
they demanded job accommodations (67.64\%) or job change (80.89\%); 55.42\% did not feel understood or supported by their company in their limitations due to migraine, although they did feel they were supported by their colleagues (63.07\%).

Conclusion: We found that preventive resources and information were deficient, and that there was little use of adaptive management options for workers with migraine in their companies.

Key Words: migraine, occupational risk prevention, occupational health

\section{Introducción}

La promoción y protección de la salud en el trabajo integra iniciativas de seguridad en el ámbito ocupacional, mejoras en salud y bienestar de las personas que trabajan. Dichas iniciativas conllevan un incremento de la productividad y menores riesgos y gastos sociales. Las encuestas poblacionales en trabajadores muestran que, tanto en lo que respecta a los riesgos para la salud que son modificables, como en las afecciones crónicas como la migraña, se observan las cargas económicas más elevadas. Ello justifica en las empresas la integración de iniciativas preventivas, con especial énfasis en las conductas de riesgo y en los problemas de salud modificables, como es el caso de la migraña(1). Esta es una enfermedad neurológica con una prevalencia estimada del 11.6\% en la población a nivel global, con pequeñas variaciones por países(2).

Es objetivo de este trabajo valorar la situación preventiva de diferentes países europeos y estimar la relación de la migraña con el trabajo y la gestión preventiva que se realiza en las empresas.

\section{Material y método}

Estudio observacional transversal a partir de una encuesta anónima auto-administrada de 32 ítems con respuesta múltiple no validada, ubicada en la web de la European Migraine and Headache Alliance (EMHA) y avalada científicamente por la Asociación Española de Especialistas en Medicina del Trabajo (AEEMT). Participaron 3.342 pacientes diagnosticados de migraña por sus médicos asistenciales y socios como tales de una asociación de pacientes europea (EHMA). Se incluyeron resultados de personas de España, Italia, Francia, Portugal, Irlanda, Reino Unido, Alemania y otros países de la Unión Europea (UE) no incluidos entre los anteriores en el diseño inicial del estudio y que respondieron a ella con posterioridad.

Fueron criterios de inclusión: haber sido diagnosticado previamente de migraña por su médico, estar trabajando en el momento de la encuesta o haberlo estado en el año previo y participar de forma voluntaria. Se recogen datos desde septiembre de 2018 hasta enero de 2019. A partir del análisis descriptivo inicial se analizaron de forma específica las respuestas correspondientes a los aspectos preventivos que vienen definidas por las preguntas 17, 20 a 23 y 30 del cuestionario: riesgos 
del puesto que desempeña (se puede marcar más de una), servicio de prevención en la empresa, servicio médico en la empresa, reconocimientos periódicos de vigilancia de la salud, repercusiones laborales de la migraña y opciones de gestión en la empresa.

Se realizó un análisis bivariante para cada opción propuesta y su relación con los distintos parámetros, presentando tablas de contingencia que muestran frecuencia absoluta (n) y porcentaje (\%) para cada cruce de variables. Según la naturaleza de las variables del cuestionario (variables categóricas), se utilizó el test chi-cuadrado o test exacto de Fisher para analizar la posible relación entre las características de la migraña y las variables. El análisis de los datos se realizó de forma independiente para cada una de las posibles respuestas.

\section{Resultados}

En este trabajo se muestran los resultados de la "Encuesta Europea Trabajo y Migraña" que se relacionan con los riesgos laborales referidos por los trabajadores que han respondido a la encuesta y que afectan a su migraña. Destacan el estrés laboral (77.65\%), uso de pantallas de visualización de datos (PVD) (63.87\%) y el ruido (36.25\%). En la comparativa por países participantes en la encuesta, se observan diferencias en los siguientes riesgos: exposición a ruido, trabajo a turnos o nocturno, estrés laboral, condiciones medioambientales, ergonomía del puesto y uso de PVD (Tabla 1). La respuesta es bastante homogénea en los dos riesgos referidos con más frecuencia: el estrés laboral y el uso de PVD. En España, destacan como más prevalentes porcentualmente: el estrés 23.9\%, el uso de PVD 19.35\% y trabajos que requieren alta atención o precisión 13.7\%. Los resultados completos de muestran en la Tabla 2.

Tabla 1: Riesgos laborales referidos por los trabajadores.

\begin{tabular}{|c|c|c|c|c|c|c|c|}
\hline \multirow[t]{2}{*}{ Riesgo } & \multicolumn{2}{|c|}{$\begin{array}{c}\text { No } \\
\text { riesgo referido }\end{array}$} & \multicolumn{2}{|c|}{$\begin{array}{c}\text { Sí } \\
\text { riesgo referido }\end{array}$} & \multicolumn{2}{|c|}{ Total } & \multirow[t]{2}{*}{$\mathrm{p}$-valor } \\
\hline & $n$ & $\%$ & $\mathrm{~N}$ & $\%$ & $n$ & $\%$ & \\
\hline $\begin{array}{l}\text { Mmc } \\
\text { Ruido } \\
\text { Químicos } \\
\text { Turnos/nocturno } \\
\text { Estrés } \\
\text { Conducción } \\
\text { Vibraciones } \\
\text { Atención/precisión } \\
\text { Maquinaria de riesgo }\end{array}$ & $\begin{array}{l}2960 \\
2128 \\
3054 \\
2824 \\
746 \\
3201 \\
3209 \\
2531 \\
3290\end{array}$ & $\begin{array}{c}88.68 \\
63.75 \\
91.49 \\
84.6 \\
22.35 \\
95.9 \\
96.14 \\
75.82 \\
98.56\end{array}$ & $\begin{array}{c}378 \\
1210 \\
284 \\
514 \\
2592 \\
137 \\
129 \\
807 \\
48\end{array}$ & $\begin{array}{c}11.32 \\
36.25 \\
8.51 \\
15.4 \\
77.65 \\
4.1 \\
3.86 \\
24.18 \\
1.44\end{array}$ & $\begin{array}{l}3338 \\
3338 \\
3338 \\
3338 \\
3338 \\
3338 \\
3338 \\
3338 \\
3338\end{array}$ & $\begin{array}{l}100 \\
100 \\
100 \\
100 \\
100 \\
100 \\
100 \\
100 \\
100\end{array}$ & $\begin{array}{c}<0.0001 \\
0.083 \\
0.005 \\
<0.0001 \\
<0.0001 \\
0.021 \\
0.085 \\
<0.0001 \\
0.497\end{array}$ \\
\hline $\begin{array}{l}\text { Condiciones } \\
\text { medioambientales }\end{array}$ & 2663 & 79.78 & 675 & 20.22 & 3338 & 100 & $<0.0001$ \\
\hline $\begin{array}{l}\text { Ergonómicos } \\
\text { PVD } \\
\text { Otros riesgos } \\
\text { Los desconozco }\end{array}$ & $\begin{array}{l}2694 \\
1206 \\
2842 \\
3248 \\
\end{array}$ & $\begin{array}{c}80.71 \\
36.13 \\
85.14 \\
97.3 \\
\end{array}$ & $\begin{array}{c}644 \\
2132 \\
496 \\
90 \\
\end{array}$ & $\begin{array}{c}19.29 \\
63.87 \\
14.86 \\
2.7 \\
\end{array}$ & $\begin{array}{l}3338 \\
3338 \\
3338 \\
3338 \\
\end{array}$ & $\begin{array}{l}100 \\
100 \\
100 \\
100 \\
\end{array}$ & $\begin{array}{c}<0.0001 \\
<0.0001 \\
0.191 \\
0,001 \\
\end{array}$ \\
\hline
\end{tabular}

Se consideran significativos valores de $p<0,005$.

aRiesgos no contemplados en los anteriores epígrafes 
Tabla 2: Distribución porcentual de los riesgos del puesto de trabajo según los distintos países participantes en el estudio.

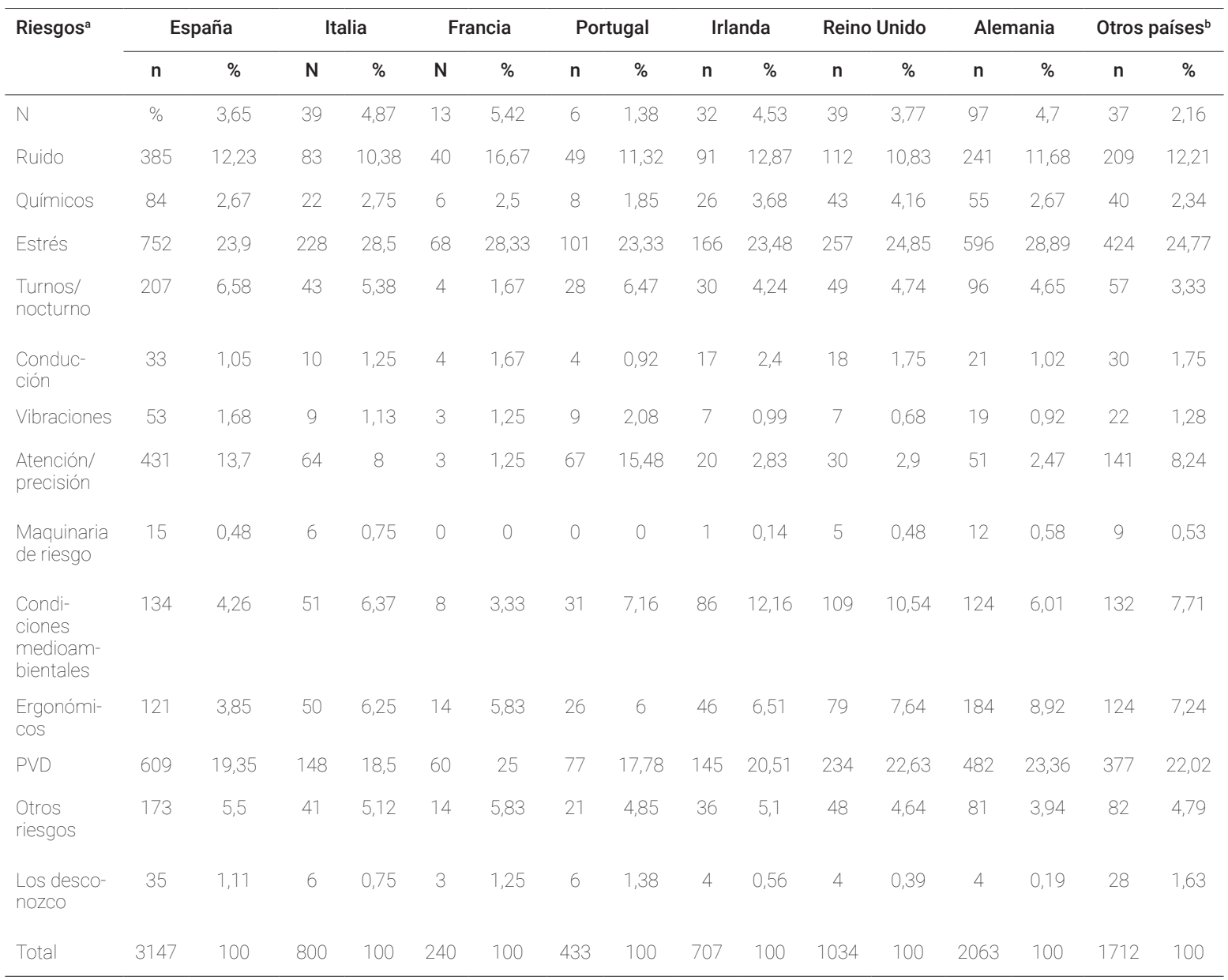

ael diseño de encuesta permite seleccionar más de un riesgo laboral

baíses no incluidos en el diseño inicial y que se incorporaron posteriormente sin especificación individualizada

En cuanto a los principales aspectos relacionados con la prevención en las empresas, se observa que un elevado porcentaje de trabajadores desconoce qué tipo de Servicio de Prevención tiene en su empresa (43.71\%) y, entre los que sí lo saben, predomina el Servicio de Prevención Propio (36.93\%). Cerca de la mitad de los encuestados refiere no disponer de Servicio Médico en su empresa (49.06\%) y, cuando lo hay, sólo el $18.12 \%$ de los casos dispone de médico a jornada completa. En conjunto, la asistencia a reconocimientos médicos (RM) de vigilancia de la salud es baja: solo el $21.27 \%$ de los trabajadores acude anualmente, el $9.12 \%$ lo hace cada dos años y el $14.66 \%$ de forma esporádica, mientras que el $29.05 \%$ de participantes no asiste nunca. El 25.9\% desconoce que se realicen RM de Vigilancia de la salud en su empresa (Tabla 3). 
Tabla 3: Aspectos Preventivos de las empresas y significación estadística en la comparativa de todos los países participantes $^{a}$

\begin{tabular}{|c|c|c|c|c|c|c|c|c|c|c|c|c|c|}
\hline \multirow{2}{*}{$\begin{array}{l}\text { Tipo de } \\
\text { servicio de } \\
\text { prevención }\end{array}$} & \multicolumn{3}{|c|}{ SPP } & \multicolumn{3}{|c|}{ SPA } & \multicolumn{4}{|c|}{ Desconocido } & \multicolumn{2}{|c|}{ total } & $\frac{\text { p-valor }}{<0.0001}$ \\
\hline & $\begin{array}{c}n \\
1221 \\
\end{array}$ & & & 64 & & 19.36 & & 1445 & & 71 & 3306 & 100 & \\
\hline $\begin{array}{l}\text { Servicio } \\
\text { médico en la } \\
\text { empresa }\end{array}$ & \multicolumn{2}{|c|}{$\begin{array}{l}\text { Jornada } \\
\text { completa }\end{array}$} & \multicolumn{2}{|c|}{$\begin{array}{l}\text { A tiempo } \\
\text { parcial }\end{array}$} & \multicolumn{2}{|c|}{ No se dispone } & \multicolumn{4}{|c|}{ Desconocido } & \multicolumn{2}{|c|}{ total } & p-valor \\
\hline \multirow{3}{*}{$\begin{array}{l}\text { Asistencia a } \\
\text { los recono- } \\
\text { cimientos } \\
\text { médicos de } \\
\text { vigilancia de la } \\
\text { salud }\end{array}$} & \multicolumn{2}{|c|}{$\begin{array}{c}\text { Asistencia } \\
\text { anual }\end{array}$} & \multicolumn{2}{|c|}{$\begin{array}{c}\text { Asistencia } \\
\text { bianual }\end{array}$} & \multicolumn{2}{|c|}{$\begin{array}{l}\text { Asistencia } \\
\text { esporádica }\end{array}$} & \multicolumn{2}{|c|}{ No asistencia } & \multicolumn{2}{|c|}{ Desconocido } & \multicolumn{2}{|c|}{ total } & p-valor \\
\hline & $n$ & $\%$ & $n$ & $\%$ & $n$ & $\%$ & $n$ & $\%$ & $n$ & $\%$ & $n$ & $\%$ & $<0.0001$ \\
\hline & 702 & 21.27 & 301 & 9.12 & 484 & 14.66 & 959 & 29.05 & 855 & 25.9 & 3301 & 100 & \\
\hline
\end{tabular}

aSe contabilizan solo las cuestiones respondidas y encuadradas en el 100\%. Se consideran significativos valores de $p<0,005$

Francia es, con diferencia, el país con mayor asistencia anual a los RM (51.16\%); la asistencia bienal es la más frecuente en Portugal e Italia y la esporádica en el bloque de otros países de la UE (no incluidos en el diseño inicial) y en Irlanda. Los resultados completos por países se muestran en la Figura 1.

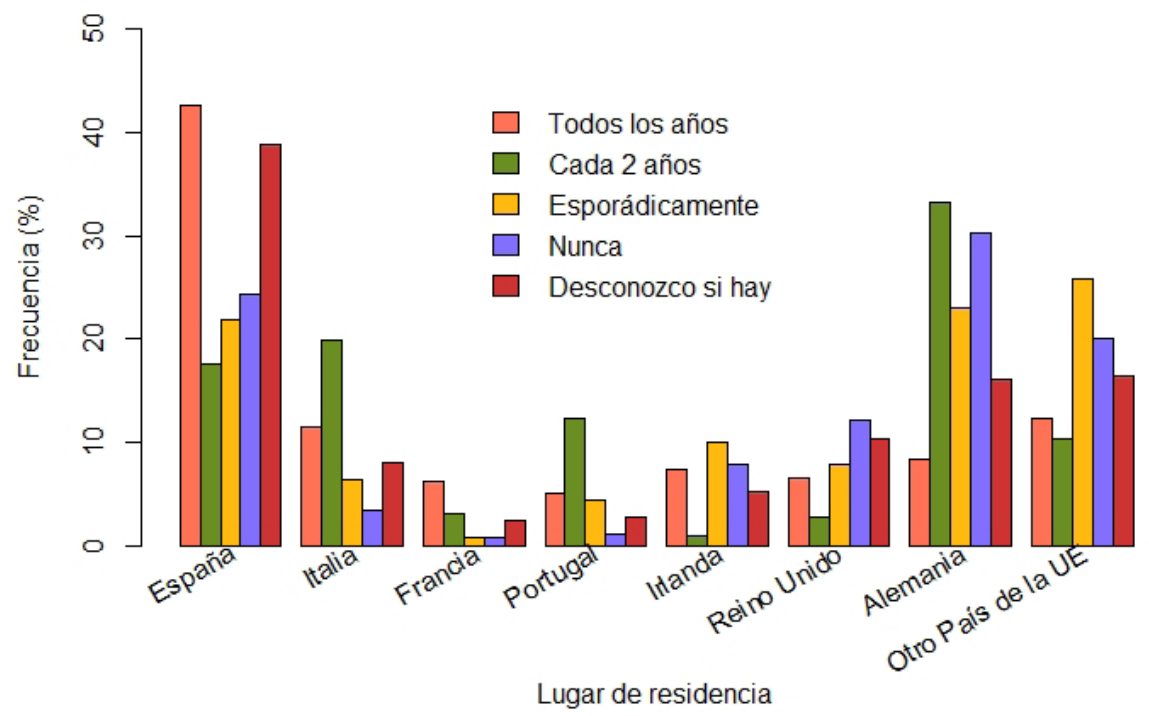

Figura 1. Reconocimientos periódicos de vigilancia de la salud en la empresa en que trabaja según los distintos países.

Con relación a las repercusiones laborales que al trabajador le ha supuesto la migraña, destaca el hecho de que la mayoría de los trabajadores (67.67\%) refiere no haber tenido problemas para acceder al trabajo, ni haber sido despedido o no haber renovado contrato por su migraña (88.29\%), sin embargo, el $42.14 \%$ de los encuestados ha tenido dificultades en su empresa por esta enfermedad, aunque 
lo más frecuente es que haya sido de forma ocasional o esporádicamente (el 33\%, y el $21 \%$ alguna vez al mes). Se observa desconocimiento del concepto de especial sensibilidad $(26,54 \%$ ) y por esta causa no han solicitado revisión con el médico del trabajo para su valoración en relación con las limitaciones por migraña (63,8\%). Los participantes en la encuesta tampoco refieren, por el hecho de padecer migraña, haber solicitado modificaciones laborales (67,64\%) o cambios de puesto $(80,89 \%)$. El $55,42 \%$ no se ha sentido comprendido ni apoyado por su empresa en sus limitaciones por migraña, pero sí por los compañeros (63,07\%), (Tabla 4).

Tabla 4: Repercusiones laborales de la migraña y significación estadística.

\begin{tabular}{|c|c|c|c|c|c|c|}
\hline \multicolumn{7}{|c|}{ Cuestiones que se plantean: } \\
\hline \multicolumn{7}{|c|}{ ¿La migraña le ha impedido acceder a algún trabajo? } \\
\hline Sí & & No & & Total & & p-valor \\
\hline N & $\%$ & n & $\%$ & $N$ & $\%$ & $<0.0001$ \\
\hline 1048 & 32.33 & 2194 & 67.67 & 3242 & 100 & \\
\hline \multicolumn{7}{|c|}{ ¿Le han despedido del trabajo o no renovado su contrato por padecer migraña? } \\
\hline Si & & No & & Total & & p-valor \\
\hline N & $\%$ & $n$ & $\%$ & $N$ & $\%$ & $<0.0001$ \\
\hline 376 & 11.71 & 2834 & 88.29 & 3210 & 100 & \\
\hline \multicolumn{7}{|c|}{ ¿Ha tenido dificultades en su empresa por padecer migraña? } \\
\hline Sí & & No & & Total & & p-valor \\
\hline N & $\%$ & $n$ & $\%$ & $N$ & $\%$ & $<0.0001$ \\
\hline 1359 & 42.14 & 1866 & 57.86 & 3225 & 100 & \\
\hline
\end{tabular}

En el caso de que haya tenido dificultades o conflicto laboral por limitaciones-pérdida de productividad para realizar adecuadamente su trabajo por padecer migraña, ¿con que frecuencia?

\begin{tabular}{|c|c|c|c|c|c|c|c|c|c|c|c|c|}
\hline \multicolumn{2}{|c|}{ Diariamente } & \multicolumn{2}{|c|}{ Semanalmente } & \multicolumn{2}{|c|}{$\begin{array}{l}\text { Alguna vez/ } \\
\text { mes }\end{array}$} & \multicolumn{2}{|c|}{ Muy esporádica } & \multicolumn{2}{|c|}{ No repercute } & \multicolumn{2}{|l|}{ Total } & \multirow{2}{*}{$\begin{array}{l}p \text {-valor } \\
0.0005\end{array}$} \\
\hline N & $\%$ & N & $\%$ & $N$ & $\%$ & $n$ & $\%$ & $n$ & $\%$ & $n$ & $\%$ & \\
\hline 164 & 5.09 & 329 & 10.21 & 683 & 21.2 & 1093 & 33.93 & 952 & 29.56 & 3221 & 100 & \\
\hline
\end{tabular}

¿Ha solicitado ser considerado como trabajador especialmente sensible por su migraña con relación al puesto de trabajo que desempeña?

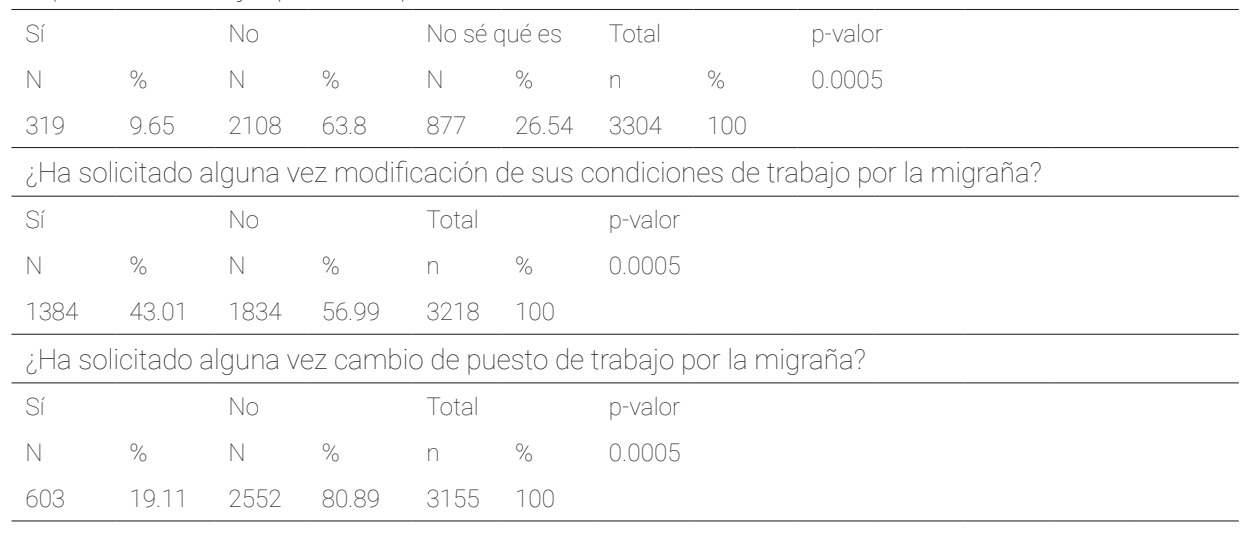




\begin{tabular}{|c|c|c|c|c|c|c|}
\hline \multicolumn{7}{|c|}{ Cuestiones que se plantean: } \\
\hline \multicolumn{7}{|c|}{$\begin{array}{l}\text { Si lo ha solicitado ¿Le han adaptado o ajustado de alguna forma el puesto de trabajo en su empresa } \\
\text { por la migraña? }\end{array}$} \\
\hline \multicolumn{2}{|l|}{ Sí } & \multicolumn{2}{|l|}{ No } & \multicolumn{2}{|l|}{ Total } & \multirow{3}{*}{$\begin{array}{l}\text { p-valor } \\
0.0005\end{array}$} \\
\hline n & $\%$ & $\mathrm{n}$ & $\%$ & n & $\%$ & \\
\hline 731 & 32.36 & 1528 & 67.64 & 2259 & 100 & \\
\hline \multicolumn{7}{|c|}{ ¿Se ha sentido comprendido y apoyado por su empresa por las limitaciones que implica la migraña? } \\
\hline $\mathrm{Si}$ & & \multicolumn{2}{|l|}{ No } & \multicolumn{2}{|l|}{ Total } & p-valor \\
\hline n & $\%$ & n & $\%$ & $n$ & $\%$ & 0.0005 \\
\hline 1420 & 44.58 & 1765 & 55.42 & 3185 & 100 & \\
\hline \multicolumn{7}{|c|}{$\begin{array}{l}\text { ¿Se ha sentido comprendido y apoyado por sus compañeros en relación a las limitaciones que } \\
\text { implica la migraña? }\end{array}$} \\
\hline \multicolumn{2}{|l|}{ Sí } & \multicolumn{2}{|l|}{ No } & \multicolumn{2}{|l|}{ Total } & p-valor \\
\hline$n$ & $\%$ & $n$ & $\%$ & $n$ & $\%$ & 0.0005 \\
\hline 2027 & 63.07 & 1187 & 36.93 & 3214 & 100 & \\
\hline
\end{tabular}

En todos los países, los trabajadores con migraña, refieren limitaciones que repercuten en su productividad, a excepción de Alemania donde se encuentra el mayor porcentaje de trabajadores en los que la migraña no representa una limitación en su rendimiento laboral (Figura 2).

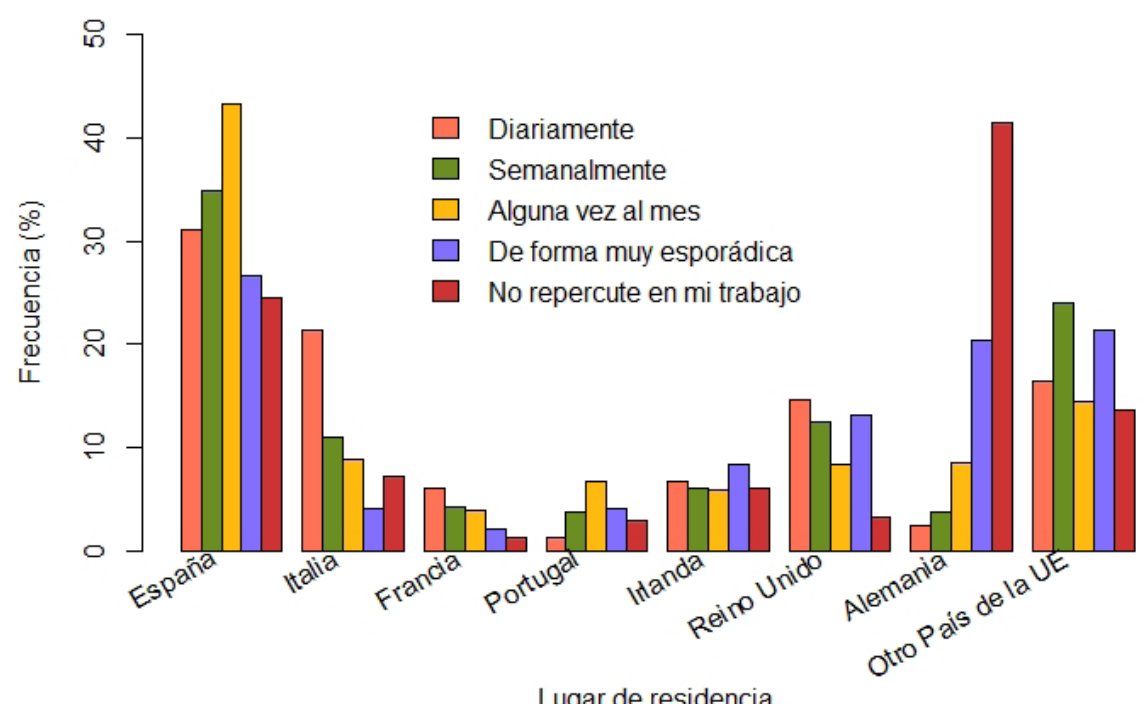

Figura 2: ¿Ha tenido dificultades en su empresa por padecer migraña? Respuesta según los distintos países. 


\section{Discusión}

La Encuesta Europea Trabajo y Migraña aporta datos de interés específicos en el ámbito de la Salud Laboral al haber sido diseñada para el mundo del trabajo y con una visión preventiva. Las publicaciones previas de este estudio muestran, respecto a las características sociodemográficas y laborales de la población que ha respondido a la encuesta, un perfil de persona que se corresponde con: mujer, de edad media, del ámbito urbano, nivel educativo medio-alto, apoyo moderado del entorno en sus crisis, que son prolongadas, de elevada frecuencia, con ambos tipos de migraña, utilizan medicación preventiva, tratamiento sintomático con triptanes, trabajan por cuenta ajena, en el sector sanitario o en la administración pública y perciben el trabajo como medio de integración social(3).

La demanda médica asistencial por migraña en Europa muestra resultados irregulares por países, siendo principalmente dirigida hacia la especialidad de Neurología y con menor participación de médicos de Atención Primaria, Médicos del Trabajo, Enfermería u otras especialidades. Se observan diferencias por edad, género y nivel cultural tanto en la demanda asistencial como en la elección de especialista. Un importante porcentaje de pacientes se automedican o no llevan ningún control médico( ${ }^{(4)}$. Los resultados referidos a los tratamientos sintomáticos utilizados para la migraña muestran gran variabilidad en cada país y se ven modificados por diferencias sociales y demográficas, siendo la edad, el género, el ámbito de residencia y el nivel cultural los factores que más condicionan el uso de cada medicación sintomática para las crisis de migraña ${ }^{(5)}$. Los resultados muestran que el uso de terapias farmacológicas preventivas en la migraña sigue siendo bajo a pesar de contar con respaldo científico, por lo que resulta imprescindible reforzar la capacitación del médico y la información al paciente, evaluando sus preferencias para mejorar la adherencia a las terapias prescritas ${ }^{(6)}$.

La migraña afecta aproximadamente al 10\% de los adultos en edad laboral y se asocia con un aumento en los costos de atención médica y pérdida de jornadas y horas de trabajo. Sin duda, buena parte de la intervención laboral debe surgir de los aspectos formativos y del conocimiento preventivo de los trabajadores, pero también de los gestores de las empresas. Así lo muestran algunos programas en países como Estados Unidos, donde la implantación de un programa educativo en el lugar de trabajo para el manejo de la migraña ha permitido impactar significativamente en la pérdida de productividad y en el absentismo generado por esta enfermedad ${ }^{(7)}$.

Nuestro estudio se ha basado en una encuesta no validada y que recopila un amplia información recogida en 32 items, de los cuales, una parte significativa no habían sido contemplados hasta el momento ya que se han basado en aspectos preventivo-laborales que no forman parte de las encuestas habituales utilizadas para esta enfermedad y que no permiten valorar estos aspectos tan directamente ligados al mundo del trabajo. Las encuestas validadas sobre migraña valoran el impacto en calidad de vida y en días perdidos por discapacidad como son: Chronic Pain Index, HImQ, Baltimore County, Headache Impact Questionnaire, Migraine 
Disability Assessment Questionnaire (MIDAS) o el cuestionario validado para el estudio del "Plan de Acción de Lucha contra la Migraña" (PALM) ${ }^{(8-17)}$.

Muchos estudios en ámbito laboral relacionados con la migraña se centran en sectores laborales específicos, como el sanitario(18). Nuestro trabajo se ha dirigido a los riesgos laborales en su conjunto más que al sector de actividad. Destaca el peso cuantitativo del estrés, el uso de pantallas de visualización de datos y el ruido como los más representativos y referenciados por los trabajadores con migraña. Los estudios afirman que la migraña es un trastorno relacionado con el estrés, lo que sugiere que puede haber hiperactividad simpática en estos pacientes, sin embargo, hay resultados contradictorios con respecto a la activación simpática en pacientes con migraña y, en general, no se encuentran diferencias significativas respecto a los pacientes-control, excepto una tendencia leve que se observa en estos pacientes a reaccionar con menos activación vagal y más actividad simpática en todas las pruebas realizadas ${ }^{(19)}$. En nuestra encuesta hay una referencia clara al impacto del estrés en la migraña, por lo que es recomendable actuar preventivamente sobre este componente de riesgo laboral. En otros trabajos, si bien se reconoce el estrés como factor de riesgo en migraña se apunta la tendencia, observada generalmente en patologías crónicas como la migraña, a desarrollar mecanismos de adaptación al estrés ${ }^{(20)}$.

El uso de PVD es otro de los aspectos contemplados en los estudios realizados con las cefaleas primarias ${ }^{(21)}$, mostrando coincidencia con los resultados obtenidos en nuestro trabajo respecto a la percepción de los pacientes en cuanto a su impacto en la migraña. La aparición de migraña se relaciona en múltiples publicaciones con actividades y condiciones laborales y de estilo de vida variadas, pero sin que pueda establecerse una clara relación causa-efecto con la severidad o frecuencia de la enfermedad. Esto ocurre con el estrés y la relajación, los productos dietéticos específicos, el ayuno, la fatiga, la luz brillante, el ruido, los cambios climáticos y medioambientales, si bien se admite que algunos o todos ellos pueden influir en alguna medida en la frecuencia y severidad de la migraña ${ }^{(22)}$. Nuestros resultados coinciden con otros previos en el bajo apoyo social que los pacientes perciben durante sus crisis de migraña ${ }^{(23)}$.

El lugar de trabajo es un espacio adecuado para realizar actividades preventivas sobre enfermedades crónicas como la migraña, que por su frecuencia y severidad repercuten personal, social y laboralmente. Nuestro estudio muestra en todos los países un elevado desconocimiento de los servicios de prevención, escaso uso de los servicios médicos en las empresas, cuando los hay, baja asistencia a los reconocimientos de vigilancia de la salud y desconocimiento o no uso de las opciones de gestión preventiva de sus empresas. Sin embargo, la literatura hace referencia al impacto favorable de estas actividades y fomenta la inclusión de un modelo de atención administrada para la migraña en los programas de salud y bienestar desde las unidades básicas de salud, con implicación de la enfermería del trabajo que permita identificar y derivar a pacientes con migraña evitando la automedicación y buscando mejor control del dolor para, de este modo, disminuir su frecuencia e intensidad y aumentar la calidad de vida(24). Nuestros resultados refuerzan lo con- 
cluido ya en años previos sobre la importancia de las condiciones de trabajo como factor de riesgo para la cefalea primaria, especialmente el estrés laboral, físico y mental, fuertemente asociado con la migraña en tareas que conllevan trabajo mental o físico intenso. La mejora de las condiciones de trabajo podría prevenir las crisis de dolor, disminuyendo el sufrimiento individual y el gasto del empleador y del sector público(25).

En nuestra encuesta los trabajadores no refieren conflictividad laboral por migraña, ni generar despidos y la única especificación problemática es la relacionada con la pérdida de productividad durante las crisis, lo que coincide con trabajos previos que incluyen este aspecto en la valoración de migraña, al igual que ocurre en otras enfermedades crónicas ${ }^{(26)}$.

Algunos trabajos han validado cuestionarios concretos que abordan el impacto de la migraña en el trabajo en términos de dificultades en habilidades generales o específicas y en cuanto a los factores que contribuyen a estas dificultades, definidos como impacto negativo en las tareas laborales y que se puede también utilizar para evaluar el equilibrio entre los efectos terapéuticos y secundarios de la medicación en la productividad laboral(27).

Los trabajadores de nuestro estudio refieren mayor comprensión de sus limitaciones por migraña por sus compañeros que por los gestores de sus empresas. Coincide esta visión con lo publicado por otros autores que hablan de rechazo o prejuicios de la sociedad hacia los pacientes con patologías crónicas como la migraña y afirman que existe un estigma en torno a la migraña, que parece ser más frecuente en pacientes con ciertas características sociodemográficas, y que relacionan con el estrés, las alteraciones del estado de ánimo y la baja autoestima. Intentar reducir estos factores podría contribuir a mejorar el control de la migraña y reducir el impacto de la enfermedad a nivel socioeconómico ${ }^{(28)}$.

Este trabajo presenta como fortalezas su tamaño muestral y la inclusión de países de la Unión Europea que sirven de comparativa con el nuestro, además de abordar aspectos preventivos y laborales poco tratados previamente. Son limitaciones de este estudio, los derivados del uso de una encuesta no validada, la subjetividad de las respuestas, la mayor participación de mujeres, la distribución no uniforme de los participantes por países, la exclusión de personas menores de 18 años o mayores de 65 (fuera de la edad laboral), la no inclusión de personas no laboralmente activas y la indefinición del grupo de países que no se incluyeron en el diseño inicial y cuyos pacientes respondedores no han podido ser diferenciados.

La intervención médica coordinada y el conocimiento de estos aspectos ocupacionales pueden proporcionar herramientas clínicamente relevantes y una optimización ocupacional y social en el uso de los recursos disponibles. 


\section{Conclusiones}

Un elevado porcentaje de trabajadores desconoce qué tipo de servicio de prevención tiene en su empresa. La mayor parte de las empresas no tienen servicio médico propio y, en los casos en los que existe, es poco utilizado por los trabajadores. Los trabajadores muestran escasa asistencia a los reconocimientos médicos de vigilancia de la salud cuando los tienen. Los trabajadores muestran poca dificultad para acceder al trabajo por migraña y no refiere haber tenido problemas de despido o renovación de su contrato, aunque sí refieren problemática laboral relacionada con menor productividad durante las crisis. El concepto de trabajador especialmente sensible no es conocido y por ello los trabajadores no demandan su valoración a las empresas, en los casos necesarios. Los participantes en esta encuesta sí habían solicitado modificaciones de sus condiciones de trabajo por migraña, pero en pocas ocasiones pidieron cambio de puesto. En general se sienten comprendidos y apoyados por sus compañeros en relación con las limitaciones por sus crisis de migraña, pero no del mismo modo por sus empresas.

\section{Agradecimientos}

A la Asociación Española de Especialistas en Medicina del Trabajo (AEEMT), a la European Migraine Headache Alliance (EMHA) y a los pacientes que han respondido esta encuesta.

\section{Financiación}

Este trabajo ha recibido financiación de la European Migraine Headache Alliance (EMHA) (Ref.: EMHA2018/02).

\section{Bibliografía}

1. Street TD, Lacey SJ. Accounting for employee health: The productivity cost of leading health risks. Health Promot J Austr. 2019;30:228-37.

2. Woldeamanuel YW, Cowan RP. Migraine affects 1 in 10 people worldwide featuring recent rise: A systematic review and meta-analysis of community-based studies involving 6 million participants. J Neurol Sci. 2017;372:307-15.

3. Vicente-Herrero MT, Ruiz de la Torre E, Reinoso Barbero L. Trabajo y migraña. Encuesta europea. Medicina Balear. 2019;34:30-7.

4. Vicente-Herrero MT, Ramírez Iñiguez de la Torre MV, Ruiz de la Torre E, Reinoso Barbero L. Migraine medical control and related variables. Results of the European Work and Migraine survey. Semergen. 2020;46:225-33

5. Vicente-Herrero MT, Ramírez Iñiguez de la Torre MV, Ruiz de la Torre E, Reinoso Barbero L. Tratamiento sintomático en migraña. Fármacos utilizados y variables 
relacionadas. Resultados de la encuesta europea sobre trabajo y migraña. Rev Soc Esp Dolor. 2020;27:178-91

6. Vicente-Herrero MT, Ramírez-Iñiguez de la Torre MV, Ruíz-de la Torre E, Reinoso-Barbero L. Preventive Treatment In Migraine. Used Drugs And Related Variables. Results Of The European Work And Migraine Survey. Rev Mex Neuroci. 2020;21:82-9.

7. Burton WN, Chen CY, Li X, McCluskey M, Erickson D, Schultz AB. Evaluation of a Workplace-Based Migraine Education Program. J Occup Environ Med. 2016 Aug;58(8):790-5.

8. Lipton RB, Stewart WF, Diamond S, Diamond ML, Reed ML. Prevalence and burden of migraine in the United States: data from the American Migraine Study II. Headache. 2001; 41: 646-57.

9. Stewart WF, Lipton RB, Liberman J. Variation in migraine prevalence by race. Neurology. 1996; 47: 52-9

10. Slater ND, Lipton RB, Stewart WF. Doctor-patient communication about migraine disability. Neurology. 1999; 52 (Suppl 2): 209.

11. Lipton RB, Stewart WF, MacGregor A. Communication of migraine disability between physicians and patients. Cephalalgia. 1999; 19: 337.

12. Stewart WF, Lipton RB, Simon D, Liberman J, Von Korff M. Validity of an illness severity measure for headache in a population sample of migraine sufferers. Pain. 1999;79:291-301

13. Stewart WF, Lipton RB, Kolodner K, Liberman J, Sawyer J. Reliability of the migraine disability assessment score in a population-based sample of headache sufferers. Cephalalgia. 1999;19:107-14.

14. Stewart WF, Lipton RB, Sawyer J. An international study to assess the reliability of the migraine disability assessment (MIDAS) score. Neurology. 1999;52:988-94.

15. Stewart WF, Lipton RB, Kolodner KB, Sawyer J, Lee C, Liberman JN. Validity of the migraine disability assessment (MIDAS) score in comparison to a diary-based measure in a population sample of migraine sufferers. Pain. 2000;88:41-52.

16. Min $Y$, Rendas-Baum R, Varon SF, Kosinski M. Validation of the Headache Impact Test (HIT-6TM) across episodic and chronic migraine. Cephalalgia. 2011;31:357-67.

17. Porta-Etessam J, López-Gil J, Matías-Guiu A, Fernández C, en representación del Comité Científico del Programa PALM. Validación de un cuestionario diagnóstico para migraña adaptado para encuesta telefónica asistida por ordenador. Neurologia. 2010;25:163-7.

18. Xie W, Li R, He M, Cui F, Sun T, Xiong J, et al. Prevalence and risk factors associated with headache amongst medical staff in South China. J Headache Pain. 2020;14;21:5. 
19. Santos IS, Griep RH, Alves MG, Goulart AC, Lotufo PA, Barreto SM, et al. Job stress is associated with migraine in current workers: the Brazilian Longitudinal Study of Adult Health (ELSA-Brasil). Eur J Pain. 2014;18:1290-7.

20. González Quintanilla V, Toriello Suárez M, Gutiérrez González S, Rojo López A, González Suárez A, Viadero Cervera R, et al. Stress at work in migraine patients: differences in attack frequency. Neurologia. 2015;30:83-9.

21. Li C, Zhang L, Zhou J, Fan Z, Wang Y, Wang X, et al. Prevalence of primary headache disorders among information technology staff in China: the negative effects of computer use and other correlative factors. BMC Public Health. 2020;20:443.

22. Rauschel V, Straube A, Süß F, Ruscheweyh R. Responsiveness of the autonomic nervous system during paced breathing and mental stress in migraine patients. J Headache Pain. 2015;16:82.

23. Chądzyński P, Kacprzak A, Domitrz W, Domitrz I. Migraine headache facilitators in a population of Polish women and their association with migraine occurrence preliminary results. Neurol Neurochir Pol. 2019;53:377-83.

24. Berry PA. Migraine disorder: workplace implications and solutions. AAOHN J. 2007:55:51-6.

25. Antonov K, Isacson D. Headache in Sweden: the importance of working conditions. Headache. 1997;37:228-34.

26. Allen D, Hines EW, Pazdernik V, Konecny LT, Breitenbach E. Four-year review of presenteeism data among employees of a large United States health care system: a retrospective prevalence study. Hum Resour Health. 2018;16:59.

27. Raggi A, Covelli V, Guastafierro E, Leonardi M, Scaratti C, Grazzi L et al. Validation of a self-reported instrument to assess work-related difficulties in patients with migraine: the HEADWORK questionnaire. J Headache Pain. 2018;19:85.

28. Martínez-Fernández A, Rueda Vega M, Quintas S, de Toledo Heras M, Díaz de Terán J, Latorre González G, et al. Psychosocial repercussion of migraine: is it a stigmatized disease? Neurol Sci. 2020;41:2207-13. 